\title{
Modeling a Large Submillimeter-Wave Observatory
}

\author{
John Z. Lou, Andy Kissil, Dave C. Redding, and Matt C. Bradford \\ Jet Propulsion Laboratory, California Institute of Technology \\ Steve Padin and David Woody \\ California Institute of Technology
}

\begin{abstract}
The 25 meter aperture Cornell Caltech Atacama Telescope (CCAT) will provide an enormous increase in sensitivity in the submillimeter bands compared to existing observatories, provided it can establish and maintain excellent image quality. To accomplish this at a very low cost, it is necessary to conduct accurate engineering trades, including the most effective segment and wavefront sensing and control approach, to determine the best method for continuously maintaining wavefront quality in the operational environment. We describe an integrated structural/optical/controls model that provides accurate performance prediction. We also detail the analysis methods used to quantify critical design trades.
\end{abstract}

\section{Introduction}

CCAT is a joint effort by Cornell University and the California Institute of Technology with the Jet Propulsion Laboratory and several other partners to conduct a conceptual design study for a 25 meter aperture submillimeter telescope for FAR-IR / submillimeter astronomy. CCAT will be sited in the Atacama Desert in Northern Chile. The baseline design is a segmented aperture, Ritchey-Chretien telescope optimized for operation at wavelengths longer than 200 microns [1]. If the design requirements can be met, the deployed CCAT will provide unprecedented high sensitivity, broad wavelength range, a wide field of view and high throughput for astronomical survey and multi-object spectroscopy, which also complements the current capabilities provided by the submillimeter astronomy facilities at Caltech Submillimeter Observatory (CSO) and ALMA.

We are building a comprehensive and realistic computer model for CCAT engineering trade studies. With CCAT cost constraints in mind, we have performed extensive component and system-level modeling and analysis to explore the most effective and low-cost options for CCAT segment alignment. Our CCAT optical model includes a complete telescope frontend (Primary segments, Secondary and Tertiary) and contains a 7-ring 210-segment model and a 6-ring 162-segment mirror model, both specified in the CCAT design study [1]. The optical model has full ray-tracing and Fourier optics analysis capabilities built in to predict wavefront error and image quality, and can include surface errors Zernike modes or any general form of surface deformations. A set of software tools were created to model segment cross-gap sensing in IR, and edge-sensor models were created to simulate CCAT segment edge-sensing measurements and generate sensitivity and control matrix for segment and wavefront control. CCAT uses CFRP subframes as the basic segment support structure, each $\sim 2 \times 2$ meter, on which 4 machined aluminum tiles are mounted. For panel thermal analysis, a finite-element model is used to generate panel surface deformations due to thermal gradient in the telescope environment. The thermally induced panel deformation will not only by itself generate telescope misalignment, but the surface deformation will also introduce edge-sensor measurement errors, and the tolerance of segment alignment algorithm on measurement errors would be small when the condition of the measurement sensitivity matrix is poor due to the existence of weak modes. An integrated modeling approach, combining thermal, structural and optical effects, is therefore needed in order to achieve a comprehensive understanding of CCAT system behavior in a realistic environment.

JPL has many years of experience in wavefront and segment sensing and control for Far-IR, IR and optical telescopes, including extensive analysis and testbed work for the James Webb Space Telescope and Keck Telescope [4][5][6] [9][10]. We are leveraging our expertise in segmented telescope sensing and control, combined with recent JPL work in edge-sensor development, to develop a comprehensive strategy for both CCAT telescope initialization and wavefront maintenance control. In the current framework, a focal or pupil plane interferometric sensing scheme, as proposed by Serabyn [8] could be used as the wavefront sensor for CCAT initial alignment, and one wavefront control option can be based on optimizing segment cross-gap measurement at the pupil plane. For CCAT wavefront maintenance, edge sensors

Ground-based and Airborne Telescopes III, edited by Larry M. Stepp, Roberto Gilmozzi, Helen J. Hall

Proc. of SPIE Vol. 7733,773326 - @ 2010 SPIE · CCC code: 0277-786X/10/\$18 - doi: 10.1117/12.856493 
are a cost-effective option for segments alignment, and JPL has developed an optical edge sensor, called the LAser Directed Displacement (LADD) sensor developed at JPL [11]. We have examined the sensitivity modes of LADD sensors for CCAT segments, and combined it with a wavefront control scheme proposed by Redding [4] to evaluate the effectiveness of segment control and robustness of sensing noise suppression of a wavefront control strategy designed for CCAT system maintenance.

This paper is organized as follows: Section 2 discusses a strategy for CCAT telescope initial alignment, including a combined cross-gap and IR sensing option which appears to be robust with sensing noise. Section 3 presents a thermal and structural model for the CCAT raft panels. Section 4 provides the detail of the LADD sensor model and discusses two segment control options for CCAT wavefront maintenance; performance results from computer simulations for the two control methods are presented and analyzed. Section 5 presents some initial CCAT integrated modeling effort, and shows from simulation how well thermal disturbances to the mirror segments can be corrected by the segment actuators; finally section 6 provides a summary.

\section{Segment Sensing and Control for CCAT Initialization}

To model and analyze several segment sensing and control approaches, a CCAT optical system model was generated using the JPL in-house optical analysis program MACOS [7]. The system parameters were drawn from the CCAT optical design specified in CCAT technical memo 47 by Padin [2]. CCAT is a Ritchey-Chretien telescope with a 25meter aperture and a system focal ratio of 6.0. Our optical model consists of the primary mirror (PM) segments as well as secondary and tertiary mirrors. The input beam is focused at the focal plane after tertiary mirror. For analysis purpose, the system wavefront is evaluated at the exit pupil located above the tertiary. In the course of CCAT feasibility design study, the PM as an active mirror had two possible segmentation layouts considered: one with 6 rings of 162 keystoneshaped segments and the other with 7 rings of 210 keystone-shaped segments. Fig. 1 shows the CCAT system model layout and the 162-segment primary. Each segment has three actuators that provide piston and tip/tilt control for positioning and orientation.
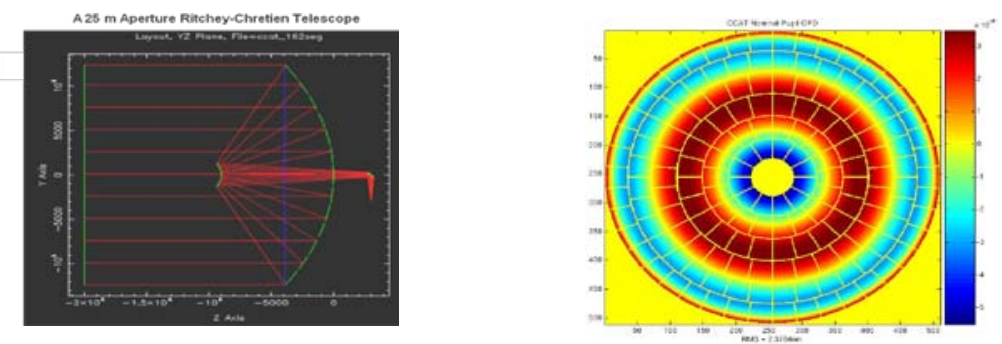

Fig. 1. CCAT optical system layout (left) and a 6-ring, 162 keystone elements PM segmentation (right)

Our analysis began with the 210-segment layout with which we explored wavefront control for initial segment alignment. Our main attention has since focused on the 162-segment configuration for studying an edge-sensing based system wavefront control approach. Edge-sensing sensitivity tends to decrease with increasing number of segments while the segment surface distortion error is obviously proportional to the segment size. With that tradeoff in mind, the 162-segment configuration is currently the CCAT baseline.

When discussing wavefront control for CCAT initialization, we want to first make it clear that for CCAT the decision is still open on finding an initialization approach that is both technically feasible and within cost constraint. One control approach we investigated and will be discussed here is based on Keck-like IR sensing techniques, and the candidate wavefront sensing approaches include focal plane shearing interferometry and pupil-plane interferometry [8], though these methods would certainly require mirrors with higher optical quality and more stable support structure than required by the current CCAT baseline and what are available today. Assuming the availability of an IR pupil wavefront sensor, we analyzed the wavefront control performance using the CCAT 210-segment configuration. In the modeling, masks are placed on each segment so that along each edge between two neighboring segments, pairs of patches are defined where light can pass through and reflect from the mirror. At the system pupil, the average ray optical path length of each patch is computed and the difference of the averaged path length between neighboring segments is computed for each patch 
pair. The optical path differences of the patch pairs, a measure of segment cross-gap, are assembled into an array, say $d g$, which can be minimized using the segment tip/tilt/piston actuators.

The tip/tilt/piston motions of 210 segments constitute a parameter space of dimension 630. If we place one patch pair along each segment edge, the total number of patch pairs is 312, so the measurement sensitivity (response) matrix would be of dimension 312 by 630. In this case, the null space of the sensitivity is very large and the system is poorly observable, implying good segment control would be difficult to achieve. If two patch pairs are used along each radial edge of neighboring segments, as shown in the left image in Fig. 2, the total number of patch pairs would be 618, which makes the system much more observable and thus controllable, as our simulation result shows.
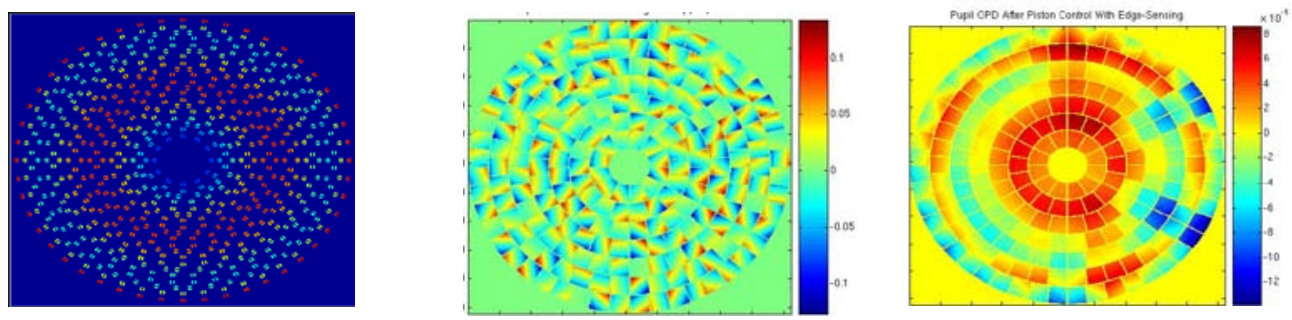

Fig. 2. Masks are placed on segments to define pairs of cross-gap patches along edges of neighboring segments. The left image shows the footprint of rays at the system pupil with masks on the segments. The middle image shows a pupil wavefront after random segment tip/tilt/piston perturbations, with OPD RMS $=33.2 \mathrm{um}$. The right image shows the controlled wavefront with RMS $=32.4 \mathrm{~nm}$. No sensing noise is added.

In the segment control simulation with 618 patch pairs, an initially perturbed segment state is generated by performing random tip/tilt/piston motions on the segments, with standard deviation of tip and tilt equal to 50 urad and that of piston equal to $50 \mathrm{um}$. The resulting pupil OPD is shown in the middle image in Fig. 2. The controller aims to minimize the measurement error vector $d w$, which is the set of averaged optical path differences of patch pairs along segment edges. The controlled pupil wavefront on the right in Fig. 2 has a residual RMS of $32.4 \mathrm{~nm}$ after $2-3$ control iterations.
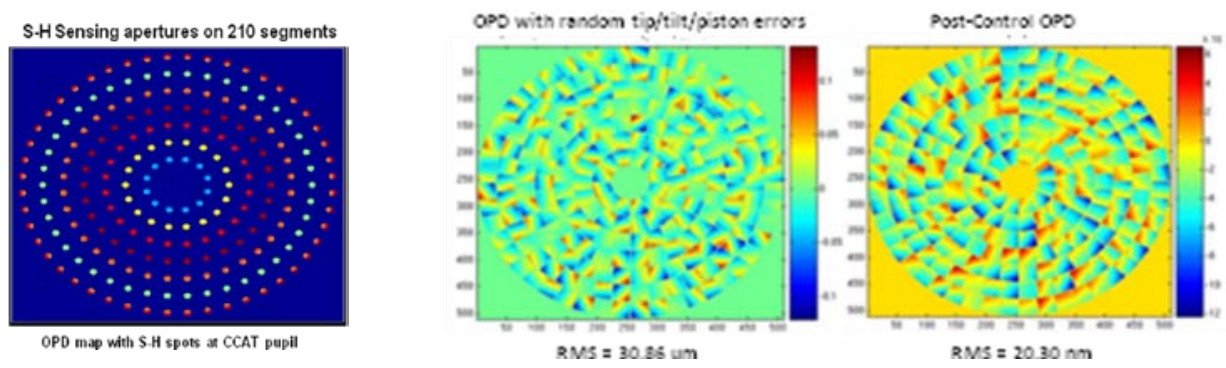

Fig. 3. The left image shows the segment S-H sensing spots at the system pupil. The middle image shows a pupil wavefront from an initially perturbed segment state with RMS of about $30.8 \mathrm{um}$, and the right image is the controlled wavefront using the combined cross-gap and S-H centroid measurement with a residual wavefront error of $20.3 \mathrm{~nm}$. Cross-gap sensing noise of $30 \mathrm{~nm}$ and S-H sensing noise of $30 \mathrm{nrad}$ are included.

When the measurement sensitivity matrix is singular, as is the case with 618 cross-gap patch pairs, the segment control process is susceptible to instability from sensing noise - a small amount of sensing errors gravely degrade the control performance. One option to get additional segment state measurement and thus constraint would be to add a ShackHartmann type sensing patch at the center of each segment, as shown in the left image of Fig. 3. The S-H sensors measure centroid motions of the S-H spots that correspond to segments tip/tilt, and produce a measurement vector $d c$ that can be used in combination with the cross-gap measurement vector $d g$ to generate a total measurement vector [ $d g$; $d c]$. Thus the measurement matrix equation can be written as [dg; $d c]=[d g d x ; d c d x]^{*} d x+d n$, where $d g d x$ and $d c d x$ are cross-gap and S-H centroid sensitivity matrices, respectively; $d x$ is the segments tip/tilt/piston state and $d n$ is the sensing noise. With the standard deviations of segment tip/tilt/piston initial errors the same as in the previous control simulation, and the standard deviation of $d n$ set at $30 \mathrm{~nm}$ for $d g$ and $30 \mathrm{nrad}$ for $d c$, the reduction of RMS wavefront error by the controller is shown in Fig. 3. 
Segment sensing and control performance is also tested in the presence of atmospheric distortion to optical phase. The measurement equation with atmospheric distortion has the general form $d m=[d g ; d c]=[d g d x ; d c d x]^{*} d x+[d g d w$; $0] * d w_{a t m}+d n$. The term $d g d w^{*} d w_{a t m}$ adds the atmospheric distortion effect to the cross-gap measurement, where $d g d w$ is basically a counting matrix of rays in each segment patch pair with entries either +1 or -1 depending on which patch the ray falls into, and $d w_{a t m}$ is the wavefront distortion due to the atmospheric turbulence. $d w_{a t m}$ is generated by a Kolmogorov phase disturbance model implemented in MACOS [7], with turbulence coherence length $r_{0}=2$ meter and atmospheric wavelength $=2 \mathrm{um}$. The control performance with atmospheric phase distortion is shown in Fig. 4. By minimizing the measurement error $d m$ with segment tip/tilt/piston actuators, the atmospheric phase distortion can be reduced from $1.5 \mathrm{um}$ to $90 \mathrm{~nm}$ without sensing error and to about $150 \mathrm{~nm}$ with $100 \mathrm{~nm}$ and 100 nrad sensing errors as shown in Fig. 4.

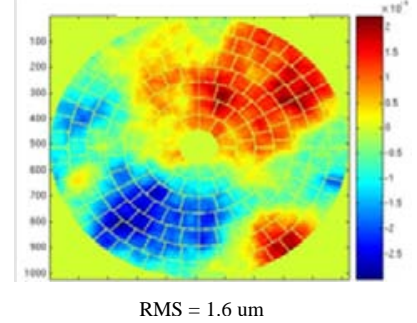

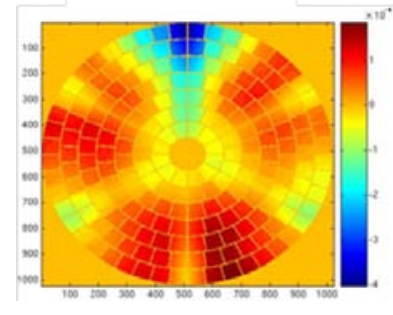

RMS $=90.1 \mathrm{~nm}$

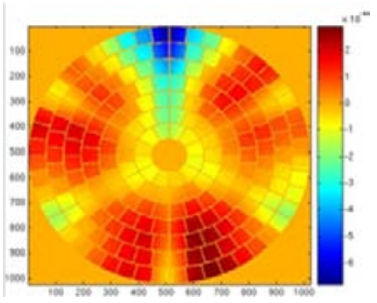

RMS $=149.8 \mathrm{~nm}$

Fig. 4. Left image shows an averaged wavefront distorted by atmospheric turbulence with $r_{0}=2 \mathrm{~m}$ and $\lambda=2 \mathrm{um}$, the middle image shows a segment tip/tilt/piston controlled wavefront of RMS $=90.1 \mathrm{~nm}$ without sensing noise, and the right image is the controlled wavefront with RMS $=149.8 \mathrm{~nm}$ where standard deviations of $100 \mathrm{~nm}$ and $100 \mathrm{nrad}$ are added to the measurements of $d g$ and $d c$, respectively.

\section{CCAT Segment Structural and Thermal Model}

The current CCAT structural model, shown in Fig. 5, is based on a preliminary design and finite element model developed by D. Woody, et al [3], and is composed of a steel axle, a CFRP primary and secondary support truss, and actuated CFRP primary segment rafts, each with a fixed array (2x2) of Aluminum reflector panels. The support truss is composed of CFRP struts connected via Invar node-balls. There are 162 CFRP primary segment rafts which are each kinematically mounted to the support truss, and thermally insulated to provide a nearly isothermal, and very stable platform.

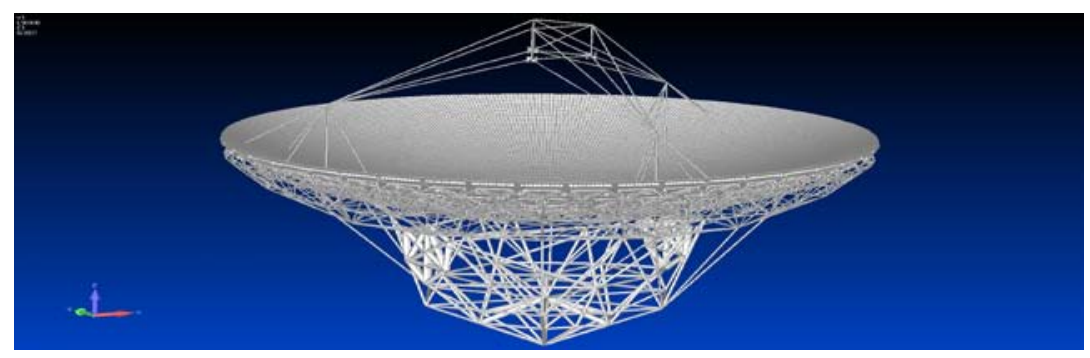

Fig. 5. CCAT Structural Finite Element Model

The CCAT NASTRAN finite element model, shown in Fig. 5, represents the support truss structure using CROD elements, and equivalent properties for the strut/node-ball assembly. The model has approximately $120 \mathrm{~K}$ nodes, which gives us under 1 million degrees of freedom: a manageable size with today's compute power, even without substructuring (e.g. super-elements). As the design matures, one would want to add more detail, including explicit joint/fitting modeling, etc., and transition to super-element modeling, to accommodate the larger model. At this stage of the design, however, we want to minimize cost, complexity and turnaround time.

The concept for the CFRP truss strut is an athermalized assembly of near-zero CTE (e.g. - $0.16 \mathrm{ppm} / \mathrm{K}$ ) composite tube, with end fittings composed of a combination of steel and negative CTE CFRP components. The FEM is currently using a nominal strut element equivalent CTE of $+0.2 \mathrm{ppm} / \mathrm{K}$, which seems to be a conservative and achievable goal, based on 
preliminary analysis of representative strut/fitting/ball detailed component models. The left image in Fig. 6 shows the cross-section of a detailed component model for a possible strut end-fitting configuration. System performance evaluation will look at sensitivity to expected strut CTE variability, based on estimated variations of material properties, as well as construction and manufacturing tolerances.

The right image in Fig. 6 shows one of the segment raft assembly models: a ring 3 segment in this case. Each raft is flexure-mounted to give a kinematic interface to the truss, and has three actuators, controlling piston, tip and tilt motion of the raft. The raft sub-base, shown in light blue, is composed of CFRP face-sheets, top and bottom, modeled with plate elements, and an aluminum honeycomb core, modeled with equivalent solid elements having orthotropic material properties. The triangular frame, shown in orange, provides support for the truss-end of the three actuators, minimizing relative motion between the actuator bases.
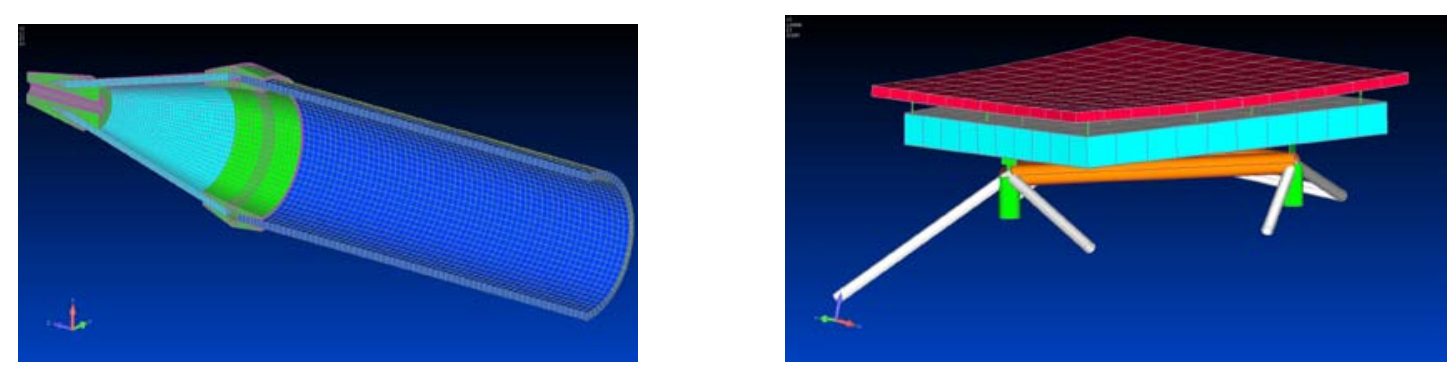

Fig. 6. Left Image: Representative Primary Support Truss Strut End-Fitting Detailed Component FEM, Right Image: Raft Assembly FEM for a Ring 3 Segment

There are four aluminum panels (2x2 array) mounted to the CFRP sub-frame: each panel mounted using five flexured stand-offs. The panel design currently modeled is an all-aluminum honeycomb sandwich construction. The invar panel stand-offs, and raft mount flexures, are modeled using beam elements.

\section{Segment Edge-Sensing and Wavefront Control for CCAT Wavefront Maintenance}

For CCAT wavefront maintenance, we consider segment position errors, due to temperature and gravity, in 6DOF rigidbody motions, which are characterized as tip, tilt, clocking, shift in X and $\mathrm{Y}$, and piston in a segment local coordinate frame. The question is how well the CCAT wavefront can be controlled given periodic edge-sensor measurements and segment corrections with segment tip/tilt/piston actuators. One option is to use the segment 3DOF actuators to minimize the edge-sensor measurement directly, a simple and seemingly logical approach. In this case, the segment controller tries to find a 3DOF segment tip/tilt/piston control vector $u_{3 d o f}$, such that the edge-sensor measurement $m$

$$
m=\left(\frac{d m}{d x}\right)_{6 \text { dof }} X_{6 \text { dof }}+\left(\frac{d m}{d x}\right)_{3 \text { dof }} u_{3 \text { dof }}+n
$$

is minimized by $u_{3 d o f}$, where $x$ is segment state and $n$ is sensing error. A potential issue with this approach is that if the sensor measurement is somewhat sensitive to segment shifts and clocking, the controller would produce segment tip/tilt/piston motions to try to reduce the sensor signals generated by segment shifts and clocking motions, but those controller-generated tip/tilt/piston motions could be detrimental to the overall segment alignment and thus could generate large wavefront error as a result. The direct control of edge sensor measurement would also be sensitive to sensor noise as we will demonstrate with our simulation result later.

A more robust approach, which was discussed in Redding's 1991 paper [6], is to use edge-sensor measurements as input to a segment state estimator. The state estimator generates an estimate of the segment 6DOF rigid-body misalignment errors, and then an estimated wavefront can be obtained from the estimated segment state. Finally a wavefront control is performed on the estimated wavefront. If the segment state estimator can capture the components of segment misalignment errors that we are concerned about - to which the wavefront is sensitive, the control on the estimated wavefront will then be able to correct those segment errors. Below we provide an outline of the mathematical framework of this segment state estimate / wavefront control procedure. Let $m$ be an edge-sensor measurement vector, $x$ the 6DOF 
segment state vector, $\frac{d m}{d x}$ the sensitivity of $x$ with respect to $m$, and $n$ the random sensing error vector, the measurement equation can then be written as

$$
m=\frac{d m}{d x} x+n
$$

$\frac{d m}{d x}$ obviously depends on the type of edge-sensor being used and on the locations of the sensors. Suppose some a priori knowledge of the statistics of initial segment alignment errors and edge-sensor measurement error is available, it can be incorporated into the segment state estimation process. Let $X_{0}$ be the covariance matrix of initial segment errors and $R$ be the covariance matrix of sensor measurement error, the segment state estimation can be obtained by minimizing the following objective function $J$ in terms of $x$.

$$
J=\frac{1}{2}\left[x^{t} X_{0}^{-1} x+\left(m-\frac{d m}{d x} x\right)^{t} R^{-1}\left(m-\frac{d m}{d x} x\right)\right]
$$

Setting $d J / d x$ to zero, we get a least-square estimate of $x$, absent from sensing noise error,

$$
x_{\text {est }}=K m=K \frac{d m}{d x} x
$$

where

$$
K=P^{-1} \frac{d m}{d x} R^{-1}, \quad P=X_{0}^{-1}+\left(\frac{d m}{d x}\right)^{t} R^{-1} \frac{d m}{d x}
$$

With sensing error included, the segment state estimate is

$$
x_{\text {est }}=K \frac{d m}{d x} x+K n
$$

The two terms in eqn. (2) clearly balance each other in the least-square procedure depending on the ratio of $X_{0}$ and $R$; the first term, a penalty term, effectively adds a soft constraint on the segment state $x$ in the estimation process. If, say, we had the knowledge that initial segment errors are small, implying the covariance error $X_{0}$ would be small and hence its inverse would be large, the first term of $J$ then puts a large penalty on $J$, which is equivalent to a constraint on segment motion estimate $x$, and would generally influence the search for a state estimation by the least-square optimizer. On the other hand, if our knowledge of initial $x$ is rather poor, a very large $X_{0}$ effectively drops the first term from $J$, and therefore there will be no constraint on $x$ when searching for a state estimate. If the sensing error is known to be large, a small $R^{-1}$ (relative to $X_{0}$ ) implies that ( $m-\frac{d m}{d x} x$ ) doesn't need be too small for the state estimate $x$, which tends to reduce noise amplification on wavefront error by the weak modes of $\frac{d m}{d x}$. With the state $x_{\text {est }}$ available, the estimated wavefront can be computed using the linear model

$$
w_{\text {est }}=w_{\text {nom }}+\frac{d w}{d x} x_{\text {est }}
$$

The estimated wavefront $w_{\text {est }}$ will then be minimized in the subsequent least-square wavefront control, which produces a controlled segment state $x_{c}$. The final performance metric of this estimate-control procedure will be the residual wavefront error $w_{c}$ that is computed by
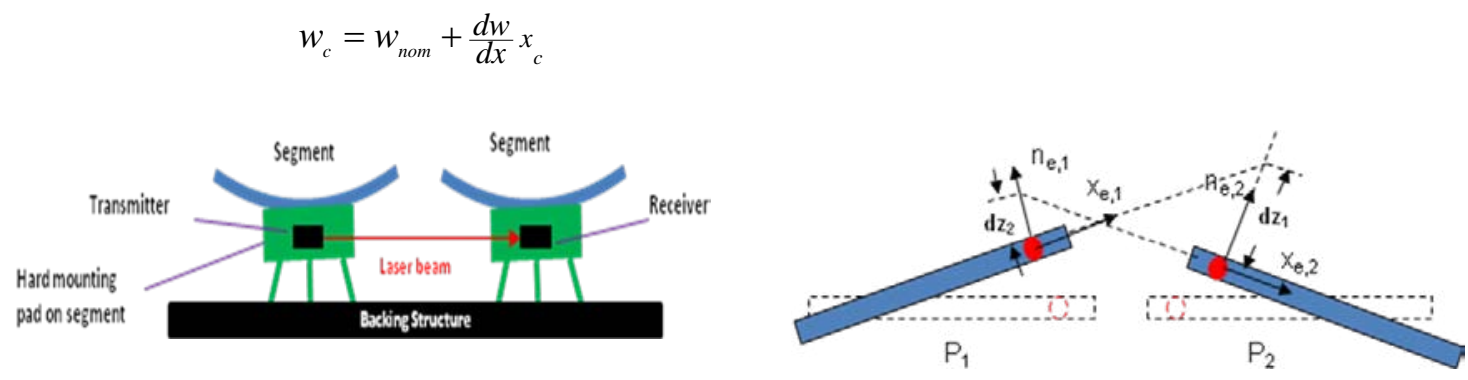

Fig. 7. LADD sensor set up schematic (left) and LADD measurement model used in the simulations (right)

With the mathematical framework of wavefront control in place, we now turn to a specific type of segment edge-sensor device, the $L A D D$ sensor. As shown in Fig. 7 with a schematic of a pair of LADD sensors situated on two adjacent 
segments, a laser beam is sent from a segment to its neighbor where a detector records a $d z$ value, its displacement from the beam's "nominal position",. The laser beam measurement is symmetric between the two neighboring segments, so for each sensing spot along a segment boundary two $d z$ values will be obtained, shown as $d z_{1}$ and $d z_{2}$ on the right in Fig. 7. Note that the laser transmitter and receiver are both fixed on the segments when they undergo 6DOF rigid-body motions, and $d z$ only measures the "vertical" displacements along $n_{e, 1}$ and $n_{e, 2}$ axes of the detector coordinate frames as shown in Fig. 7. Because $d z$ only measures vertical shift of the beam in this case, it is a 1-D sensor and is insensitive to segment lateral motions.

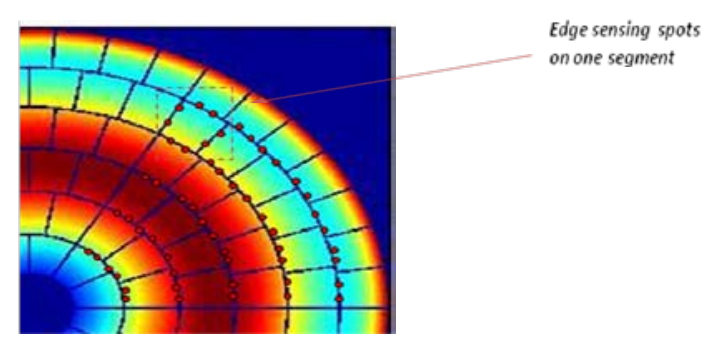

Fig. 8. LADD sensing spot distribution on segments, illustrated with segments in the $0-60^{\circ}$ quadrant of PM. Each sensing spot represents a pair of LADD sensors. Two sensing spots are placed along each radial edge, and all sensing spots along ring edges are shown in the segment quadrant.
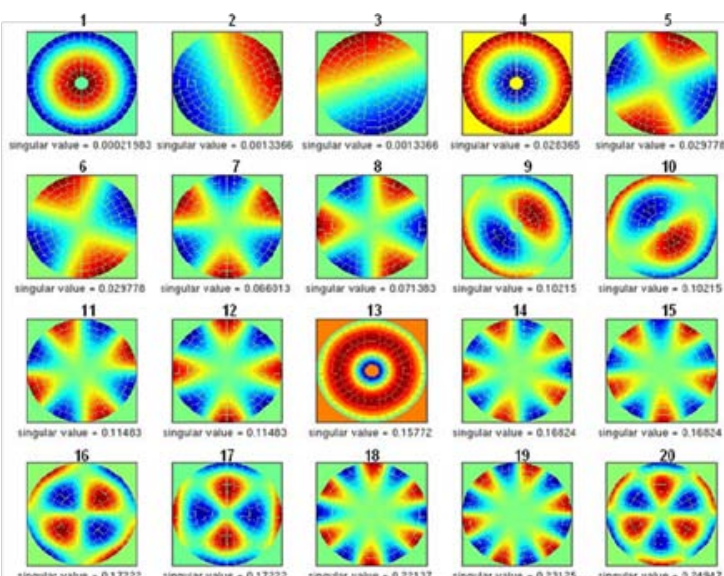

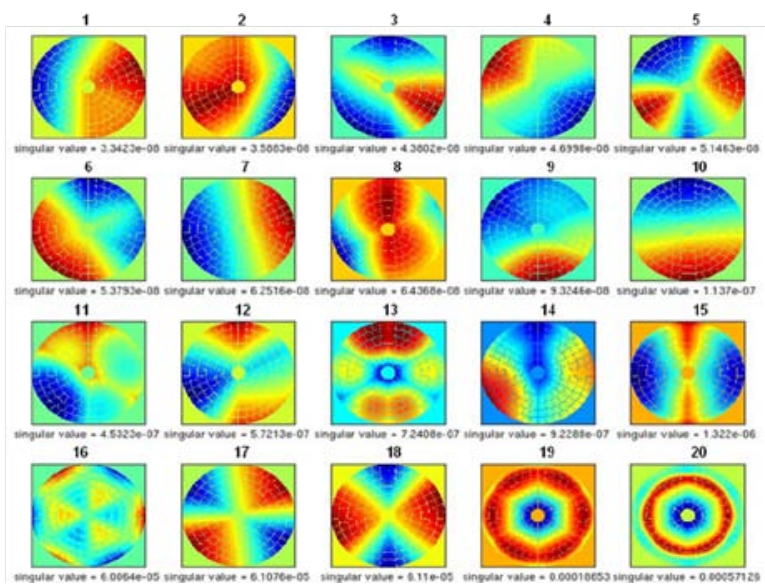

Fig. 9. LADD sensor eigenmodes. Shown are the 20 weakest modes of 3DOF sensitivity matrix on the left, and those of $6 \mathrm{DOF}$ sensitivity matrix on the right. Eigenmode maps are generated by applying the corresponding eigenvectors to the mirror surface.

Fig. 8 shows the distribution of LADD sensors on CCAT segments with a total of 624 sensing pairs. To better understand the performance of wavefront control schemes presented above, it is helpful to take a look at how the eigenmodes of the edge-sensor sensitivity matrix $d m / d x$ could impact the stability of control performance when sensor noise is present. From measurement eqn (4), assuming $X_{0}$ is very large and $R$ is an identity matrix for the ease of discussion, then $K=\left(\frac{d m}{d x}\right)^{+}$(+ is matrix pseudo-inverse), so the segment state estimate can be written as

$$
x_{\text {est }}=\left(\frac{d m}{d x}\right)^{+} m+\left(\frac{d m}{d x}\right)^{+} n=\left(\frac{d m}{d x}\right)^{+} m+\sum_{i=1}^{k} s_{i}^{-1}\left(u_{i}, n\right) v_{i}
$$

where $s_{i}$ are the singular values and $v_{i}$ are the right eigenvectors of measurement sensitivity $\mathrm{dm} / \mathrm{d} x$. The last sum in eqn (5) shows that weak modes of the sensitivity matrix, corresponding to small singular values $s_{i}$, will amplify sensor noise significantly. Since the LADD device measures the relative shifts between a pair of segments, it is clearly oblivious of any type of global motions of the mirror. Segment 6DOF rigid-body motions are defined in segment local coordinate frames in our model. When only segment tip/tilt/piston motions are considered, the 3DOF segment motions cannot achieve any exact global motion of the mirror when the mirror is not flat, but they can form mirror shapes that approximate global or low-order mirror modes that cannot be effectively detected by LADD sensor, such as global tilt and focus modes. With 6DOF segment motions, there are clearly six singular modes in the LADD sensitivity corresponding to each of the six global rigid-body motions of the mirror. Fig. 9 shows the 20 weakest modes of 3DOF 
and 6DOF LADD sensitivity matrices. The 3DOF mode maps exhibit Zernike mode-like patterns, and the weakest modes, with singular values of order $1 \mathrm{~d}-04$, include focus and tilt modes as expected. The weakest modes of $6 \mathrm{DOF}$ maps, instead of having exactly six modes of zero singular values, contain several modes of order $1 \mathrm{~d}-08$, which seem to suggest a "mixing" of weak modes on the weak end of the 6DOF sensitivity spectrum due to computational inaccuracies including those from the singular values computational scheme. The 6DOF LADD sensitivity modes are relevant in our case since it is those weak modes that could have an impact on the stability and performance of wavefront control.

It can be shown the controlled wavefront residual $w_{c}$ is largely attributed to the wavefront estimation error $d w$, which in turn is the result of segment estimation error. In fact, $w_{c}$ and $d w$ can are related by the following

$$
w_{c}=d w+\frac{d w}{d x} \cdot x_{e}-\frac{d w}{d x} A\left(\frac{d w}{d x_{u}}\right)^{+} \cdot w_{e}=d w+\left(I-\frac{d w}{d x} A\left(\frac{d w}{d x_{u}}\right)^{+}\right) \cdot w_{e}
$$

where $w_{e}$ is the estimated wavefront, $A$ is a projection from segment 6DOF motion space to the 3DOF tip/tilt/piston space. The second term on the right-hand side of eqn. (6) is basically the wavefront error caused by segment clocking and XY shifts, which are not controlled, but that part of wavefront is only about 0.2 um in current simulations.

We now present and discuss computer simulation results of CCAT LADD sensing and segment control using the two approaches described above: one is the direct minimization of LADD sensor measurement, and the other is the procedure of segment state estimate and wavefront control shown above. For all the results presented in this section, a set of 6DOF random perturbations, comprising segment tip/tilt/clocking/shift X and $\mathrm{Y}$, and piston in a segment local frame, are applied to each segment, with standard deviation of tip/tilt/clocking set at 100 microradian, and that of shift $\mathrm{X} / \mathrm{Y}$ and piston set at 100 micron, uniformly distributed with zero mean. With the perturbed segment state, LADD measurements are made through our computer model to generate a measurement vector $\mathrm{m}$. With direct sensor measurement control, the 3DOF controller simply uses the pseudo-inverse of the tip/tilt/piston sensitivity matrix $d m / d x_{3 d \text { of }}$ to generate the control vector $u_{3 \text { dof }}=-\left(d m / d x_{3 d o f}\right)^{+} m$ for segment alignment, where ' ()$^{+}$' is the pseudo-inverse operator. In the case of wavefront control, an estimated segment state is produced with the 6DOF sensitivity matrix using the estimation scheme described above. An estimated pupil wavefront is then created using the estimated segment state; a wavefront control step is then applied to the 3DOF tip, tilt and piston of all segments to minimize the estimated wavefront error, which produces a controlled segment state. The performance of the wavefront controller is evaluated by computing the true residual wavefront error with the controlled segment state.
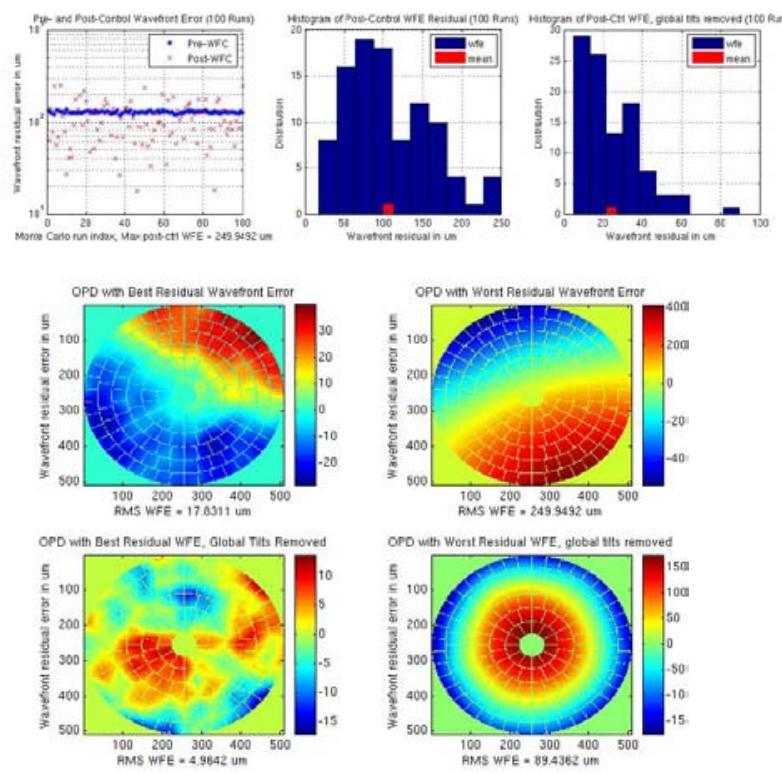

Fig. 10. 100-run Monte Carlo simulation of 3DOF direct control of LADD measurements with [-1, 1] um uniformly distributed sensing noise. The plots on the left side show the control performance with all modes of the 3DOF response matrix included, and the plots on the right side show the control performance with the three weakest rigid-body modes of the 3DOF control matrix removed. 
Fig. 10 shows the performance of 3DOF direct minimization of LADD measurements. Shown are the results of a 100run Monte Carlo simulation with LADD sensing noise at $[-1,1]$ um uniformly distributed. The effect of sensing noise on the residual wavefront error as amplified by the weakest modes of the 3DOF sensitivity matrix is clearly seen. When all modes are included in the 3DOF control, the residual wavefront error can reach as large as 89 um from the 100 runs. When the three weakest rigid-body modes are removed from the control matrix, the largest wavefront residual is significantly improved to just fewer than $14 \mathrm{um}$.

With the wavefront control approach, Fig. 11 shows the estimated wavefront of an initially perturbed wavefront. The estimation error of segment state is dominated by a global tilt component in the initial segment errors in this particular case. The 6DOF LADD sensitivity, which is used by the state estimation scheme, has exactly six singular modes corresponding to six mirror global motions, which are completely unobservable by the LADD sensors. It is expected that the estimated segment state and therefore the estimated wavefront would not be able to capture any such global motions of the mirror. The last image in Fig. 11 indicates the existence of the global tilt component in the initial wavefront, and such a global tilt is largely missed in the estimated wavefront the middle image in Fig. 11. After minimizing the estimated wavefront with a 3DOF segment control, the controlled segment state has the large global tilt component left that is reflected in the controlled wavefront as shown in the middle column of images in Fig. 12. With the global tilt removed from the controlled wavefront residual, there is about 2.5 um RMS left in controlled wavefront with a noisefree sensor, mostly astigmatism as shown in the right image of first row in Fig 12. This wavefront residual shows the extent of wavefront reduction achievable with the current sensor model and the 3DOF actuator control to correct 6DOF uncorrelated random segment misalignment errors.
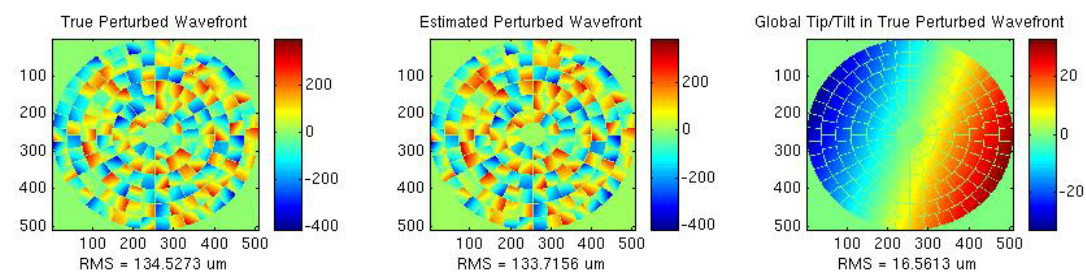

Fig. 11. Initial wavefront error as a result of random segment perturbations applied before segment control. The left image shows the true wavefront map for an initially perturbed segment state; the middle image is the estimated wavefront used in wavefront control, and the right image shows the global tilt component in the true initial wavefront.
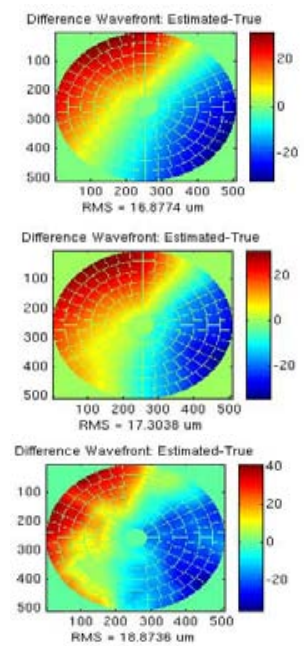
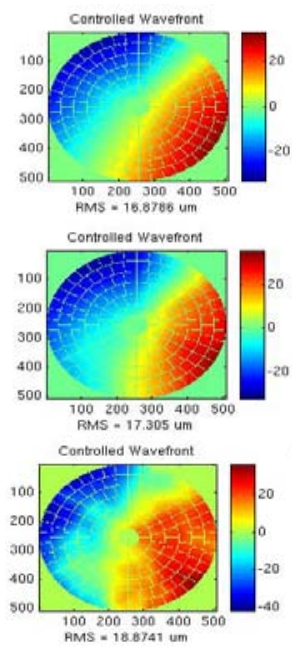
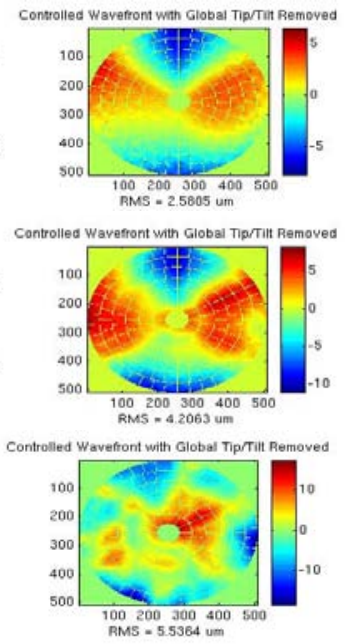

Fig. 12. Single-run LADD sensing and wavefront control simulation: the result without sensing noise is shown in images on the first row, that with $[-100,100] \mathrm{nm}$ uniformly distributed sensing noise is shown on the second row, and that with $[-1,1]$ um uniformly distributed sensing noise is shown on the last row. The wavefront controller cannot reduce any segment state errors to which the LADD sensors are insensitive, e.g. a global tilt. The first column shows the wavefront estimation errors; the second column shows the post-control residual wavefront errors, and the third column shows the residual wavefront errors with global tilts removed. 
The weak modes in the 6DOF sensitivity, as shown in Fig. 9, could cause large wavefront error from the controller when sensor noise is present. With the 6DOF LADD sensitivity, there are six global modes corresponding to the six mirror independent rigid-body motions that are completely unobservable by the edge sensor, so, in principle, the controlled wavefront residual would be sensitive to LADD sensing noise if the weak modes act directly on the noise containing those weak modes. With the wavefront control scheme we use, however, properly chosen parameters in $X_{0}$ and $R$, can effectively damp the impact of weak modes on segment state estimation error when sensor error is large. The logical choices of $X_{0}$ and $R$ values of course should be relevant to our a priori knowledge of initial segment errors and sensor errors, respectively. Fig. 12 shows the result of wavefront control simulations when $100 \mathrm{~nm}$ and 1 um random sensing errors are added, and it shows the increase of controlled wavefront residual with the increase in sensor error is quite limited.

Monte Carlo run results in Fig. 13 show the worst residual wavefront at more than 14 um RMS when LADD sensing noise is set at a more realistic level of 1 um uniformly distributed, and all 6DOF sensitivity modes are included, which is quite encouraging considering all the near singular modes in the 6DOF sensitivity matrix shown in Fig. 7. In comparison to the performance of direct control of LADD measurement when all modes are included as shown in Fig. 10, the wavefront control scheme clearly exhibits the capability of suppressing the effect of sensing noise amplification by the weak modes of the 6DOF sensitivity matrix.

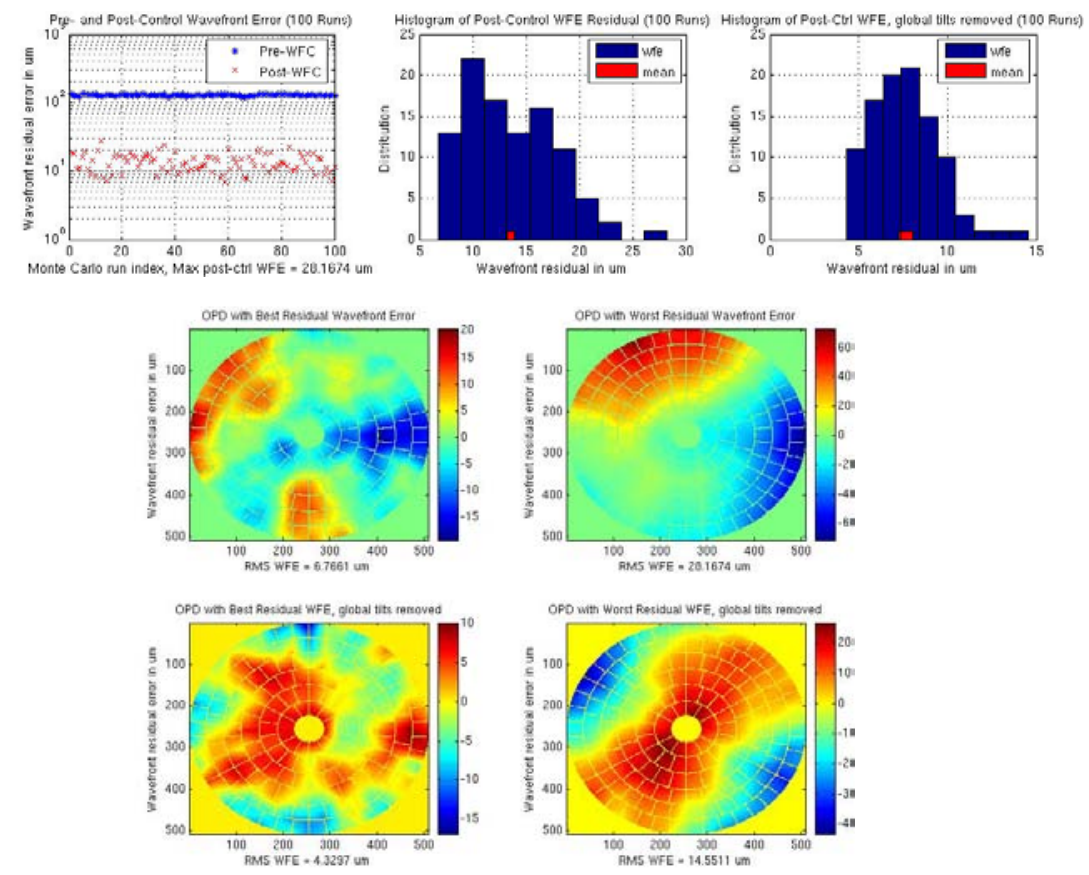

Fig. 13. 100-run Monte Carlo simulation of segment state estimation and wavefront control, with all 6DOF sensitivity eigenmodes included and LADD sensing noise at $[-1,1]$ um uniformly distributed. Best and worst wavefront residuals are shown.

The LADD measurement as depicted in Fig. 7 is a 1-D sensing model, in which only the vertical displacement of the laser beam displacement on the adjacent segment is accounted for. Such a measurement model is clearly quite insensitive to segment lateral motions along segment edges. An interesting question is whether a $2 D$ LADD measurement model, which adds the measurement of laser beam displacement along segment edges as shown in Fig. 14, would improve the segment control performance for either of the two control schemes discussed above. Our intuition would be that more measurements could be beneficial to the control performance. Fig. 14 also shows the 20 weakest eigenmodes of the 3DOF 2D LADD sensitivity matrix with singular values two orders of magnitude larger than the 1D case shown in Fig. 9. With the wavefront control scheme, the 2D LADD sensing does provide some improvement in the controlled wavefront residual as a comparison of results shown in Figs. 13 and 15 shows, which could be due to a better segment state estimate when using 2D LADD sensing. With the direct control of LADD measurement, however, the controlled wavefront turns out to be far worse than that of using 1D LADD sensing. This seemingly surprising result 
may reflect an important difference between LADD sensing signal and wavefront signal due to segment 6DOF motions. Wavefront signals due to segment tip/tilt/piston are completely decoupled from segment shift $\mathrm{x} / \mathrm{shift} \mathrm{y} /$ clocking motions, whereas LADD sensing signals due to segment tip/tilt/piston are to some extent coupled with segment shift $\mathrm{x} / \mathrm{shift}$ $\mathrm{y} /$ clocking motions. With wavefront control, a 6DOF segment state estimate tends to provide reasonable characterization of segment tip/tilt/piston motions, and the subsequent 3DOF

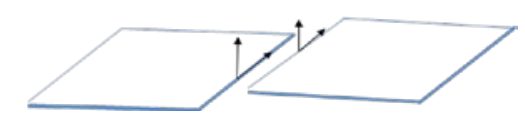

Fig. 14. 2D LADD sensing: laser beam displacement is projected onto vertical and tangential axes of local coordinate frames of neighboring segments, as shown above, to generate two measurement values. The 20 weakest eigenmodes of the corresponding 3DOF sensitivity matrix are shown on the right.
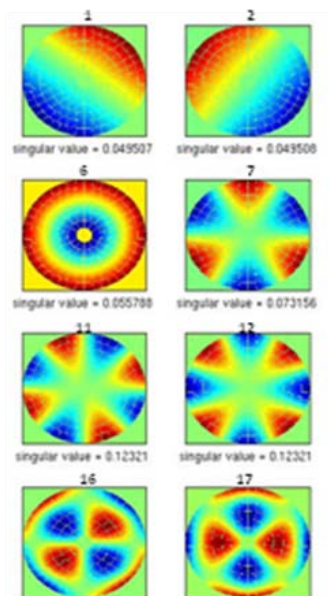
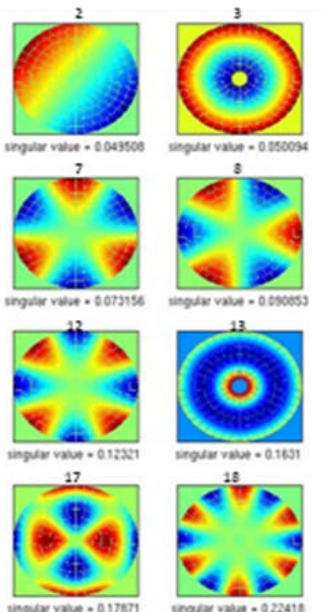
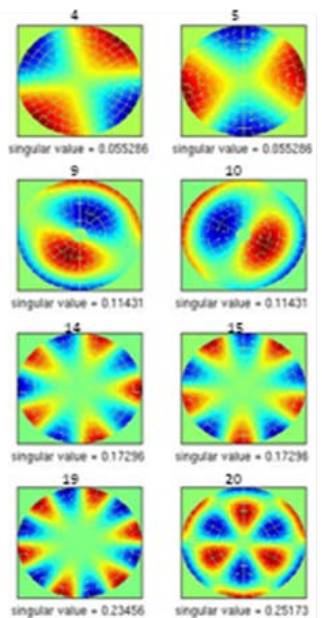

wavefront control will correct most of those segment tip/tilt/piston errors without trying to compensate segment shifts and clocking errors since they are both decoupled and do not have much footprint on wavefront error anyway. With 3DOF direct control of LADD measurement, the controller tries to reduce measurement errors caused by segment shifts and clocking using segment tip/tilt/piston due to the coupling in LADD signal, and the resulting segment tip/tilt/piston motions could further misalign the segments and generate very large wavefront error.
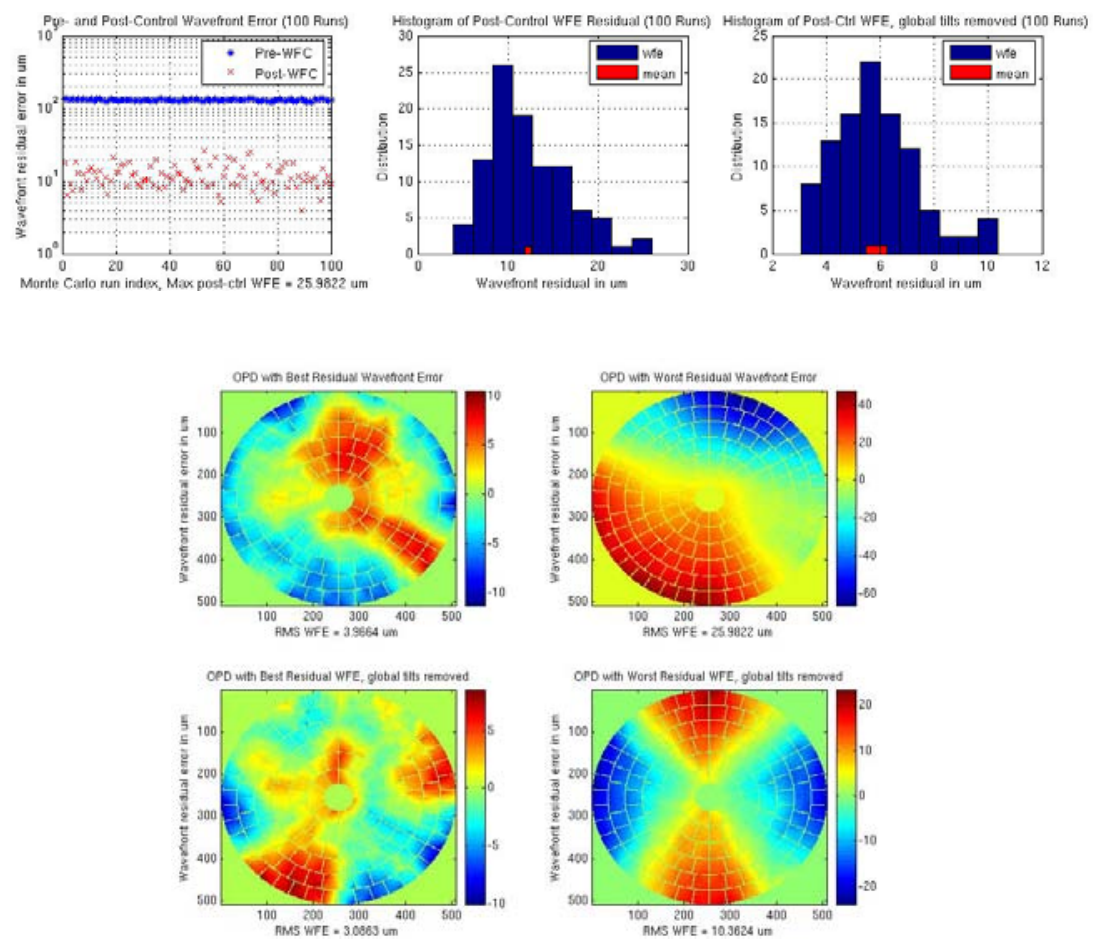

Fig. 15. 100-run Monte Carlo simulation of segment state estimation and wavefront control using 2D LADD sensing, with all modes of 6DOF sensitivity matrix included and LADD sensing noise at $[-1,1]$ um uniformly distributed. 


\section{CCAT Integrated Modeling with Thermal and Gravity Induced Segment Errors}

The simulation results in the previous section assume 6DOF initial segment disturbances that are artificially generated with a zero-mean uniform distribution. Even though the Monte Carlo simulations have provided us very useful insights on the performances of both the LADD sensor and the wavefront controller, we want to see how this framework works with segment deformations due to real thermal and gravitational environment changes. Here we present some very preliminary simulation results. Fig. 17 shows the CCAT wavefront error obtained by applying segment deformations from a +1C soak temperature change. The segment deformations are computed with a finite element model created in the NASTRAN software, and the deformation can be decomposed into a segment rigid-body motions component plus a pure segment surface figure change in the out-of-surface direction. We can see from Fig. 17 that the rigid-body motions component is the dominating contributor to the system wavefront error, which can be controlled with the segment 3DOF actuators. Fig. 18 shows the wavefront control result with the segment rigid-body motions caused by the $+1 \mathrm{C}$ soak temperature, where the 2D LADD sensing is used and $[-1,1]$ um of uniformly distributed LADD sensing noise is included.
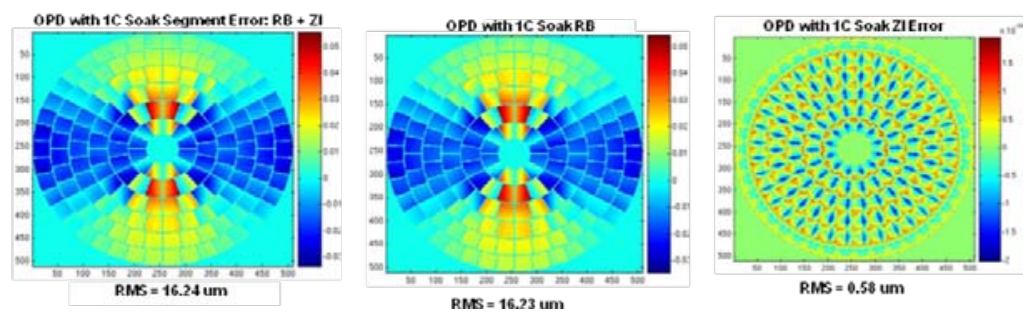

Fig. 17. CCAT wavefront error with segment deformations from $+1 \mathrm{C}$ Soak temperature. Left image shows the wavefront after the segment deformations are applied, the middle image shows the wavefront with only segment rigid-body motions of the total deformation, and the right image shows the wavefront with only segment pure shape changes.
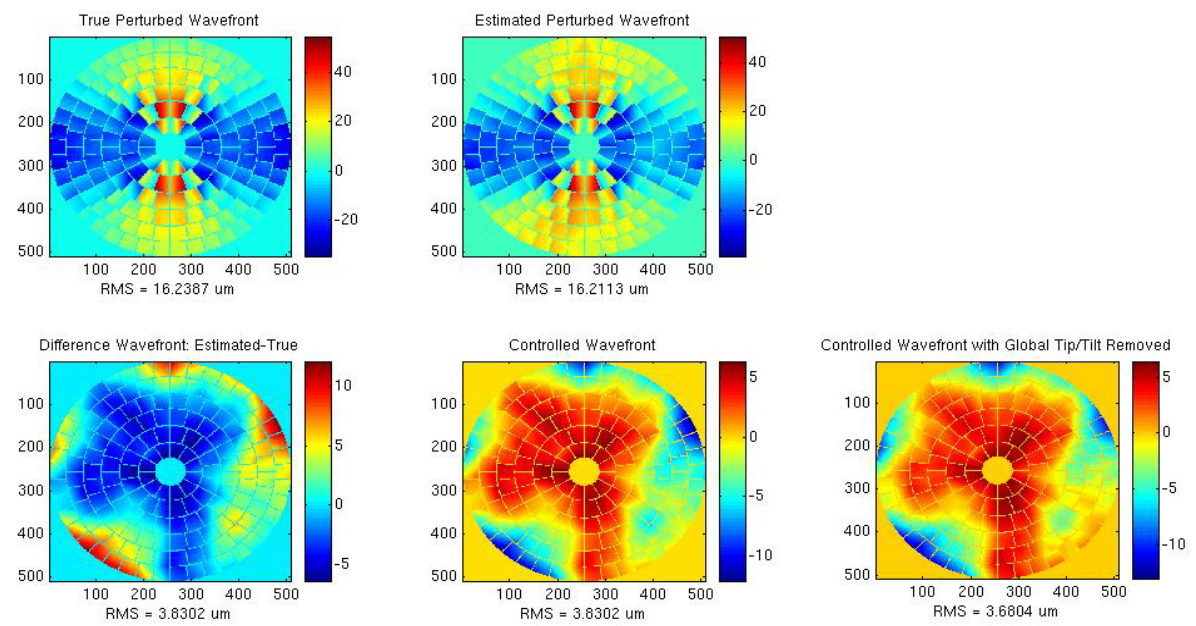

Fig. 18. Wavefront control with $+1 \mathrm{C}$ soak segment deformation. The initial segment disturbances, top left image, include only the rigid-body motions of the segments. Sensing noise of $[-1,1]$ um uniformly distributed is included.

\section{Summary}

We presented a comprehensive CCAT system model, with optical, structural and thermal components for enabling realistic engineering trade analysis, and we described segment sensing and wavefront frameworks for CCAT system initial alignment and operational wavefront maintenance. Extensive computer simulation results were presented to demonstrate the performance of our approaches. Using cross-gap IR sensing with Shack-Hartmann sensing for segment tilt control, excellent wavefront control performance can be achieved. Using a simple model of the LADD edge sensor 
and a wavefront control scheme, we demonstrated wavefront control at the few um level, even with sensor noise at 1 micron. Similar performance of wavefront control was achieved for the case with $+1 \mathrm{C}$ soak temperature segment errors.

The authors wish to thank Eri Cohen and Scott Basinger for their help in our CCAT work, and Mitch Troy and Dan MacDonald for their input on their previous work on Keck and CCAT analysis. The work described in this paper was carried out at the Jet Propulsion Laboratory, California Institute of Technology, under a contract with the National Aeronautics and Space Administration.

\section{References}

[1] Cornell Caltech Atacama Telescope (CCAT) Feasibility and Concept Design Study, Final Report, January, 2006

[2] S. Padin, CCAT Optical Design for $1^{\circ}$ FOV, CCAT Tech Memo \#47, October, 2009

[3] D. Woody, Conceptual Design for the CCAT Primary and Tipping Structure, Ver. 2.1, CCAT Tech Memo \#41, August, 2009

[4] D. Redding, B. Breckenridge, Ken Lau, G. Sevaston, M. Levine and S. Shaklan, Segmented Mirror Figure Control for a Space-Based Far-IR Astronomical Telescope, SPIE Vol. 1489, Structures Sensing and Control, 1991 / 201

[5] D. Redding, F. Shi, S. Basinger, D. Cohen, J. Green, A. Lowman, C. Ohara, Wavefront Sensing and Control for Large Space Optics, IEEE AC paper \#1720, 1-8-2003

[6] J. Lou, D. Redding, N. Sigrist, Y. Zhang, and S. Basinger, JWST On-Orbit Multi-Field Wavefront Control with a Kalman Filter Optical State Estimator, Optical Modeling and Performance Predictions II. Edited by Kahan G., Mark A. Proceedings of the SPIE, Vol. 5867, pp. 232-245, 2005

[7] D. Redding, Modeling and Analysis of Controlled Optical Systems Program (MACOS) Manual, Version 2.80, May, 1999

[8] E. Serabyn, T. Phillips and C. Masson, Surface Figure Measurements of Radio Telescopes with a Shearing Interferometer, Applied Physics, Vol. 30, No. 10, April 1991

[9] M. Troy, G. Chanan, E. Sirko and E. Leffert, Residual Misalignment of the Keck Telescope Primary Mirror Segments: Classification of Modes and Implementations for Adaptive Optics, SPIE Conference on Advanced Technology Optical/IR Telescopes VI, SPIE Vol. 3352, 0277-786X, 1998

[10] D. MacDonald, D. Woody, C. Bradford, R. Chamberlin, M. Dragovan, S. Radford, T. Sebring, J Zmuidzinias, \& P. Goldsmith, Cornell Caltech Atacama Telescope Primary Mirror Surface Sensing and Controllability, Proc. SPIE 7012, 2008

[11] A. Abramovici, Electro-Optic Measurement of CCAT Segment-Segment and Primary-Secondary Relative Motions. JPL Internal Document, 2009 